\title{
Plastic and Superionic Helium Ammonia Compounds under High Pressure and High Temperature
}

\author{
Cong Liu, ${ }^{1}$ Hao Gao, ${ }^{1}$ Andreas Hermann, ${ }^{2}$ Yong Wang, ${ }^{1}$ Maosheng Miao, ${ }^{3}$ Chris J. Pickard,,${ }^{4,5}$ \\ Richard J. Needs, ${ }^{6}$ Hui-Tian Wang, ${ }^{1}$ Dingyu Xing, ${ }^{1}$ and Jian Sun $\circledast^{1, *}$ \\ ${ }^{1}$ National Laboratory of Solid State Microstructures, School of Physics and Collaborative Innovation \\ Center of Advanced Microstructures, Nanjing University, Nanjing, 210093, China \\ ${ }^{2}$ Centre for Science at Extreme Conditions and The School of Physics and Astronomy, The University of \\ Edinburgh, Peter Guthrie Tait Road, Edinburgh EH9 3FD, United Kingdom \\ ${ }^{3}$ Department of Chemistry and Biochemistry, California State University Northridge, \\ Northridge, California 91330-8262, USA \\ ${ }^{4}$ Department of Materials Science \& Metallurgy, University of Cambridge, \\ 27 Charles Babbage Road, Cambridge CB3 OFS, United Kingdom \\ ${ }^{5}$ Advanced Institute for Materials Research, Tohoku University 2-1-1 Katahira, \\ Aoba, Sendai, 980-8577, Japan \\ ${ }^{6}$ Theory of Condensed Matter Group, Cavendish Laboratory, \\ J.J. Thomson Avenue, Cambridge CB3 OHE, United Kingdom
}

(Received 25 November 2019; revised manuscript received 25 February 2020; accepted 10 March 2020; published 9 April 2020)

\begin{abstract}
Both helium and ammonia are main components of icy giant planets. While ammonia is very reactive, helium is the most inert element in the universe. It is of great interest whether ammonia and helium can react with each other under planetary conditions, and if so, what kinds of structures and states of matter can form. Here, using crystal structure prediction methods and first-principles calculations, we report three new stable stoichiometries and eight new stable phases of $\mathrm{He}-\mathrm{NH}_{3}$ compounds under pressures up to $500 \mathrm{GPa}$. These structures may exhibit perovskitelike structures for $\mathrm{HeNH}_{3}$ and $\mathrm{He}_{2} \mathrm{NH}_{3}$, and a host-guest crystal structure for $\mathrm{He}\left(\mathrm{NH}_{3}\right)_{2}$. Superionic states are found in all these $\mathrm{He}-\mathrm{NH}_{3}$ compounds under high pressures and temperatures in which the hydrogen atoms are diffusive while the nitrogen and helium atoms remain fixed. Such dynamical behavior in helium ammonia compounds is quite different from that in helium water compounds, where weakly interacting helium is more diffusive than stronger bound hydrogen. The lowdensity host-guest phase of space group $I 4 \mathrm{~cm}$ is found to be stable at very low pressures (about $3 \mathrm{GPa}$ ) and it enters into a plastic state, characterized by freely rotating ammonia molecules. The present results suggest that plastic or superionic helium ammonia compounds may exist under planetary conditions, and helium contributes crucially to the exotic physics and chemistry observed under extreme conditions.
\end{abstract}

\section{INTRODUCTION}

Helium is the second most abundant element in the universe, and it occupies a large fraction of the atmospheres of Uranus and Neptune. Although helium is generally considered to be unreactive, several groups have reported stable helium compounds under high pressure. For instance, it can react with metals [1,2], metal oxides, sulfides, or fluorides [3-5], in which the insertion of helium atoms

\footnotetext{
* Corresponding author. jiansun@nju.edu.cn

Published by the American Physical Society under the terms of the Creative Commons Attribution 4.0 International license. Further distribution of this work must maintain attribution to the author(s) and the published article's title, journal citation, and DOI.
}

significantly modifies the long-range Coulomb interactions [5]. In addition, helium can react with inert gases [6-8], nitrogen [9,10], water [11-13], and ammonia [14], where it exhibits negligible charge transfer and plays a role in increasing the internal pressure.

Since the pioneering work of predecessors [15-17], considerable effort has been devoted to studying superionic states in hot dense molecular compounds, which are characterized by almost free diffusion of protons through fixed oxygen or nitrogen sublattices. Several theoretical [18-25] and experimental studies [26-30] have investigated the constituents of celestial bodies, which has led to theoretical models for planetary interiors that may exist within the "hot ice" layers of Uranus and Neptune. The hot ice is composed of water, ammonia, and methane under extreme pressures up to several megabars and temperatures up to several thousand kelvin, and is below the atmospheres of hydrogen and 
helium. Systematic computational investigations of these mixtures have achieved significant advances [31-36]. For instance, ammonia was predicted to react with water and form a series of compounds (with compositions ranging from $4: 1$ to $1: 2$ ) along the isentropes of Uranus and Neptune [33]. Bethkenhagen et al. [37] studied the 1:4:2 ternary mixture of ammonia, water, and methane, which is close to their solar abundance ratios, and constructed a new adiabatic model of Uranus. These studies of the mixtures in the hot ice layer have shed light on the understanding of these icy planets. This layer is likely to interact with the constituents of the upper planetary atmospheres; therefore, it is essential to understand how the molecular ices react with hydrogen or helium at high-pressure and high-temperature conditions. For instance, a recent study reported unusual chemical reactions between ammonia and hydrogen under those conditions [38]. The exotic ionic mobility behavior seen in superionic states is mirrored in applications in material science, where it greatly enhances the performance of lithium-based battery materials [39-41] and copper-based thermoelectric materials $[42,43]$.

Apart from superionic states in hot dense molecular ammonia, the plastic phase in ammonia, in which protons rotate around the fixed nitrogen atoms, has been reported to emerge below the superionic pressure range [27]. The plastic phase and rotational motion in water have been studied under high pressure [44-46]. Very recently, Li et al. [47] proposed that the plastic phases of materials with rotational disorder may be used as a new refrigeration agent, which absorbs or releases a large amount of energy when the systems enter or leave the plastic region through tuning of pressure.

Both helium and ammonia are major components of icy giant planets, but whether they can form new stable compounds under pressure and whether plastic and superionic states can exist in these compounds are still open questions. Also motivated by our previous study of the multiple superionic states in helium water compounds [13], we aim to explore the formation, structural evolution, and dynamic properties of helium ammonia compounds under high-pressure and high-temperature conditions. We have studied the helium ammonia system using crystal structure searching methods and have found eight new stable phases, some of which exhibit interesting host-guest and perovskitelike structures. We also found interesting plastic and superionic states in these compounds, which behave very differently from the helium water system.

\section{RESULTS}

We have explored the crystal structures in helium ammonia compounds below $500 \mathrm{GPa}$ using variablecomposition structure prediction methods in conjunction with density functional theory (DFT) total energy calculations. A structure is considered to be thermodynamically stable if its enthalpy of formation is negative relative to either elements or other potential compounds. We used the most stable solid phases of the related elements or compounds at the pressure range we studied to calculate the formation enthalpy. As the convex hulls shown in Fig. 1(a) and the phase diagram shown in Fig. 1(b), we found three stable compositions and eight new stable structures in the pressure range studied. These structures belong to three different stoichiometries: $\mathrm{He}\left(\mathrm{NH}_{3}\right)_{2}$, $\mathrm{HeNH}_{3}$, and $\mathrm{He}_{2} \mathrm{NH}_{3}$. We have checked the stability of the newly predicted phases against different exchangecorrelation functionals including different van der Waals (vdW) corrections, hybrid functionals, and the random phase approximation (RPA), as shown in Figs. S1-S3 in Supplemental Material [48], which all demonstrate that the results are qualitatively independent of functional choice. Phonon calculations indicate that the above structures are all dynamically stable, as shown in Fig. S4 of Supplemental Material [48]. The stable pressure range of the phases of each composition are shown in Fig. 1(b), and the pressure-induced phase transition sequences are as follows:

$$
\begin{aligned}
& \mathrm{He}\left(\mathrm{NH}_{3}\right)_{2}: \stackrel{3 \mathrm{GPa}}{\longrightarrow} \mathrm{I} 4 \mathrm{~cm} \stackrel{34 \mathrm{GPa}}{\longrightarrow} \mathrm{NH}_{3}+\mathrm{HeNH}_{3} \stackrel{155 \mathrm{GPa}}{\longrightarrow} \mathrm{Fmm} 2 \\
& \stackrel{165 \mathrm{GPa}}{\longrightarrow} \mathrm{Pbcm} \stackrel{300 \mathrm{GPa}}{\longrightarrow} \mathrm{Cm} \stackrel{450 \mathrm{GPa}}{\longrightarrow} \text { Ama2, } \\
& \mathrm{HeNH}_{3}: \stackrel{28 \mathrm{GPa}}{\longrightarrow} \mathrm{Pnma} \stackrel{165 \mathrm{GPa}}{\longrightarrow} P 2_{1} 2_{1} 2_{1} \text {, } \\
& \mathrm{He}_{2} \mathrm{NH}_{3}: \stackrel{355 \mathrm{GPa}}{\longrightarrow} P 2_{1} / c \stackrel{390 \mathrm{GPa}}{\longrightarrow} R 3 m \text {. }
\end{aligned}
$$

It should be noted that there are several energetically competitive structures for these type of molecular crystals. For instance, the enthalpies of Pbcn and $C m c 2_{1} \mathrm{He}\left(\mathrm{NH}_{3}\right)_{2}$ structures are very similar to those of the $I 4 \mathrm{~cm}$ phase at $10 \mathrm{GPa}$. A $P$ ca $2_{1} \mathrm{He}\left(\mathrm{NH}_{3}\right)_{2}$ structure has an enthalpy close to that of the Fmm $2 \mathrm{He}\left(\mathrm{NH}_{3}\right)_{2}$ phase at $100 \mathrm{GPa}$. A Pna2 $2_{1}$ $\mathrm{He}\left(\mathrm{NH}_{3}\right)_{2}$ structure is only about $1 \mathrm{meV} /$ atom higher than the $\mathrm{Pbcm} \mathrm{He}\left(\mathrm{NH}_{3}\right)_{2}$ phase at $300 \mathrm{GPa}$, while a Ima2 $\mathrm{He}\left(\mathrm{NH}_{3}\right)_{2}$ structure is only about $2 \mathrm{meV}$ /atom higher in enthalpy than the $\mathrm{Cm} \mathrm{He}\left(\mathrm{NH}_{3}\right)_{2}$ phase at $400 \mathrm{GPa}$. The lattice parameters for all the competitive structures are listed in Table I in the Supplemental Material [48].

Interestingly, we found that helium ammonia compounds with helium/ammonia ratios of $1: 1$ and $2: 1$ are inclined to form perovskitelike structures in which each nitrogen atom resides within an octahedron formed by six hydrogen atoms, which are connected to the nitrogen atoms via three covalent bonds and three hydrogen bonds, as shown in Fig. 1(c). Because of the different bond length between the covalent and hydrogen bonds, the ammonia octahedra are highly distorted. In $P n m a \mathrm{HeNH}_{3}$, half of the voids are empty and the other half are doubly occupied, while the voids in $P 2_{1} / c$ $\mathrm{He}_{2} \mathrm{NH}_{3}$ are all doubly occupied due to the increased helium atom density, as can be seen in Fig. S5 of the Supplemental Material [48]. According to their enthalpies, our predicted 


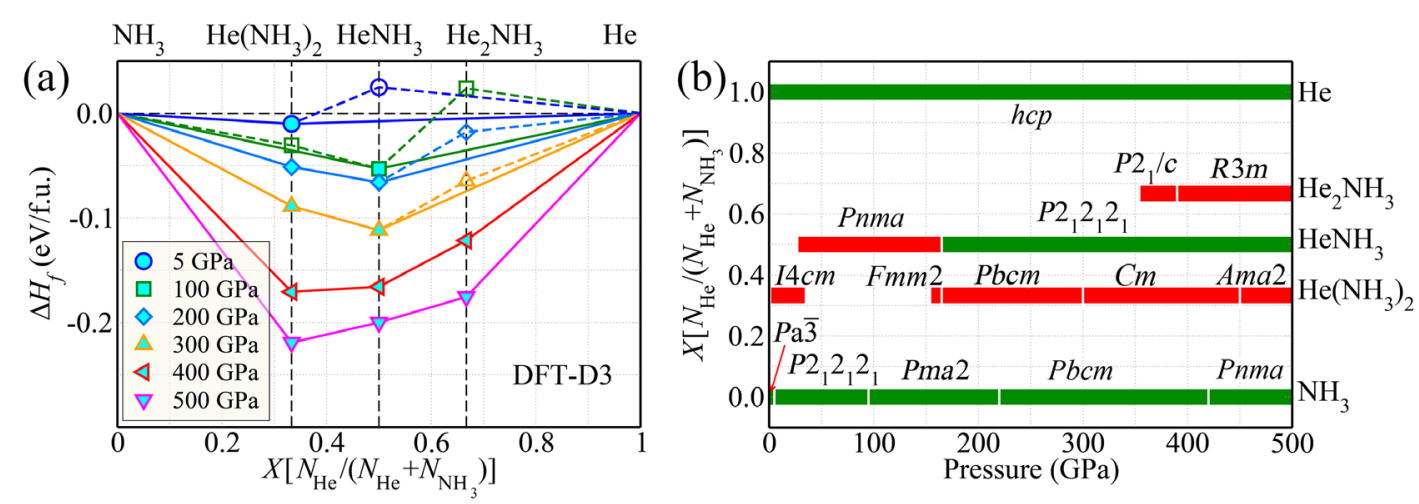

(c)
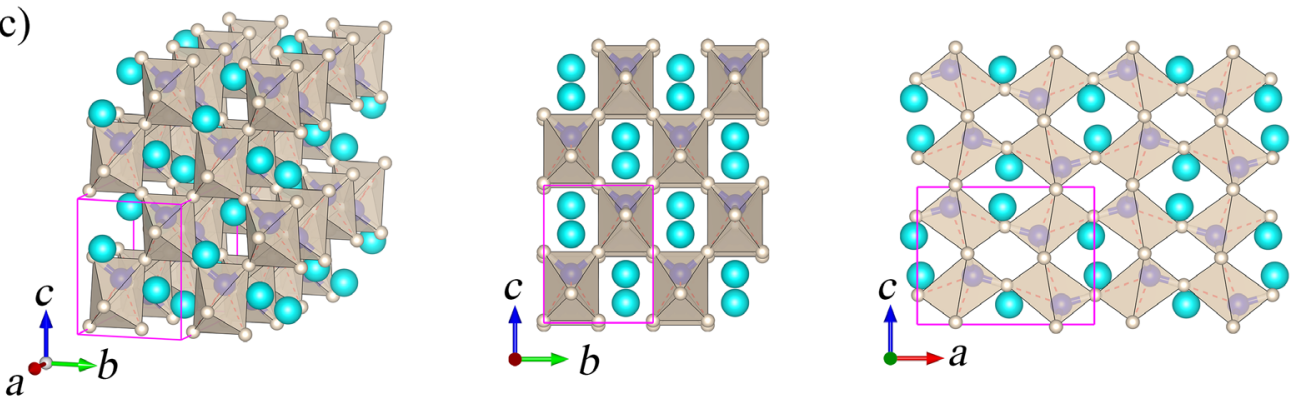

(d)

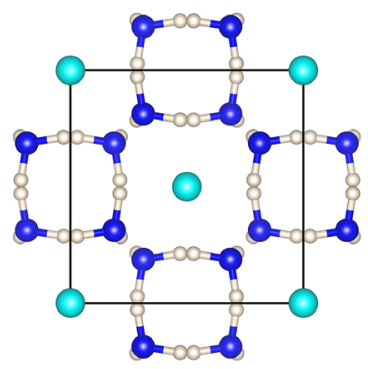

(e)

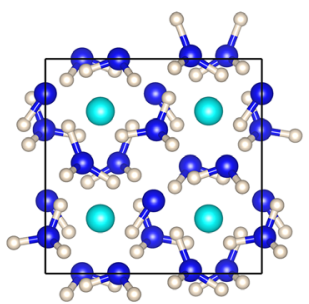

(f)

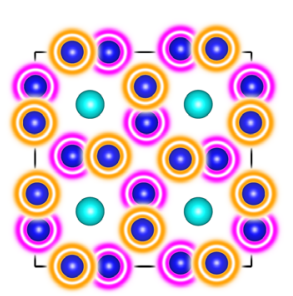

FIG. 1. Energetics of the He- $\mathrm{NH}_{3}$ system and crystal structures of the most interesting stable compounds. (a) Convex hulls for formation enthalpies $\left(\Delta H_{f}\right.$, with respect to pure $\mathrm{He}$ and $\left.\mathrm{NH}_{3}\right)$ at different pressures, calculated with the PBE + DFT-D3 functional [49]. (b) Pressure-composition phase diagram of the $\mathrm{He}-\mathrm{NH}_{3}$ phases discovered in this work (red bars), together with the previously known phases (green bars) up to $500 \mathrm{GPa}$. (c) Typical perovskite $\mathrm{HeNH}_{3}$ crystal structure of the Pnma phase at $100 \mathrm{GPa}$. (d)-(f) Typical crystal structures of host-guest phases in $\mathrm{He}\left(\mathrm{NH}_{3}\right)_{2}$. (d) the $I 4 \mathrm{~cm}$ phase at $3 \mathrm{GPa}$ and (e) the $A m a 2$ phase at $500 \mathrm{GPa}$. (f) Representation of the host-guest structure of the $\mathrm{He}\left(\mathrm{NH}_{3}\right)_{2}$ structures. Nitrogen atoms are arranged in two layers highlighted by yellow and magenta. Blue, gray, and cyan balls represent $\mathrm{N}, \mathrm{H}$, and $\mathrm{He}$ atoms, respectively.

Pnma phase $\mathrm{HeNH}_{3}$ is more stable than the previously reported $P 22_{1} 2_{1}$ phase [14] at pressures below $165 \mathrm{GPa}$, and it extends the stability range of $\mathrm{HeNH}_{3}$ down to $28 \mathrm{GPa}$.

Different from perovskitelike structures, all compounds in the $\mathrm{He}\left(\mathrm{NH}_{3}\right)_{2}$ stoichiometry belong to a host-guest prototype. As shown in Figs. 1(d)-1(f), looking along one axis, all of the nitrogen atoms are arranged in four- and eight-membered rings, and the helium atoms are distributed at the center of the eight-membered rings. In particular, the $I 4 \mathrm{~cm}$ phase becomes stable at quite low pressures (about $3 \mathrm{GPa}$ ). At higher pressures, the ammonia molecules in $\mathrm{He}\left(\mathrm{NH}_{3}\right)_{2}$ compounds are ionized by compression and form various chemical units such as $\mathrm{NH}, \mathrm{NH}_{2}, \mathrm{NH}_{3}, \mathrm{NH}_{4}$, and even long $\mathrm{N}-\mathrm{H}$ chains. More crystal structures of $\mathrm{He}\left(\mathrm{NH}_{3}\right)_{2}$ of this type can be found in Fig. S6 of Supplemental Material [48]. From our band structure calculations, both at PBE-D3 [49] and hybrid PBE0 level of theory [50], all of the helium ammonia compounds are insulating with wide band gaps, as shown in Figs. S7 and S8 [48]. The $I 4 \mathrm{~cm} \mathrm{He}\left(\mathrm{NH}_{3}\right)_{2}$ structure has a tunable band gap and an abnormal pressure dependence with a sharp increase in the band gap of around $0.064 \mathrm{eV} / \mathrm{GPa}$ over the pressure range 3-34 GPa. Recently, Liu et al. [34] carried out experimental and theoretical studies on water ammonia mixtures at extreme conditions and found that the intermediate products can be geometrically protected and thus survive for long times, which may also be a challenge for the future experimental verifications of our prediction on pure helium ammonia compounds.

To study the dynamical properties of the predicted helium ammonia compounds, we start from the $\mathrm{HeNH}_{3}$ composition, which is stable in a relatively large and 
continuous pressure range. We performed extensive $a b$ initio molecular dynamics (AIMD) simulations based on the Born-Oppenheimer approximation within the pressure range of 100-500 GPa up to the melting temperature. Diffusion coefficients $(D)$ were calculated for the nitrogen, hydrogen, and helium atoms as calculated from the averaged mean-square displacements (MSD) to monitor different types of atomic motions. More details about the methods can be found in Sec. V. This allowed us to distinguish the solid, plastic, superionic, and fluid phases.

First, we analyzed the atomic trajectories from simulations at the initial pressure of $100 \mathrm{GPa}$ for the Pnma $\mathrm{HeNH}_{3}$ phase. We calculated the averaged MSD [Figs. 2(a) and 2(b)] and compared the atomic motions in trajectories [Figs. 2(e) and 2(f)] along one axis of the simulation box. We found that the atoms oscillate around the equilibrium positions at $500 \mathrm{~K}(D=0)$, while hydrogen becomes diffusive within the static nitrogen and helium frameworks at $2000 \mathrm{~K}\left(D_{\mathrm{N}}=D_{\mathrm{He}}=0, D_{\mathrm{H}}>0\right)$. This clearly differentiates two different states: the solid phase $(500 \mathrm{~K})$ and the superionic phase $(2000 \mathrm{~K})$. Furthermore, we analyzed the atomic motions in such phases in terms of the radial distribution function (RDF), as shown in Fig. S9(a) of the Supplemental Material [48]. By heating to $2000 \mathrm{~K}$, each of the RDFs related to hydrogen became more liquidlike, while the RDFs that are not related to hydrogen remained solidlike.

Inspired by the temperature-induced features in helium ammonia compounds discussed above, we expanded the pressure range to explore the superionic region up to about $500 \mathrm{GPa}$ with a pressure increment of $100 \mathrm{GPa}$, as shown in Fig. 3(a). Every colored symbol corresponds to an independent simulation to avoid memory effects, and the pressures and temperatures were obtained as statistical averages. According to the averaged MSD, RDF, and the averaged structure, the final states of these simulations were classified into four types: solid (blue squares), plastic (dark green diamonds), superionic (cyan triangles), and fluid (orange circles). Dashed lines were fitted from the boundary points of each type of atomic motion which divide the phase diagram into four regions: the solid, plastic, superionic, and fluid phases. On the boundary between the superionic and fluid phases we found that helium atoms begin to become diffusive together with superionic hydrogen within a fixed nitrogen sublattice, as shown in Fig. S11 [48]. However, the diffusion coefficient of helium is almost 2 orders of magnitude lower than that of hydrogen, which is very different from superionic helium water compounds in which the helium atoms diffuse faster than the protons [13]. Since NVT simulations tend to overestimate melting temperature, such a helium diffusion regime in helium ammonia compounds may actually indicate an approach of the melting temperature; however, the possible superionic helium region should be very small if there is any. Compared with pure ammonia, the insertion of helium greatly extends the superionic region to much higher temperatures above $200 \mathrm{GPa}$; see Fig. 3(a). While the isentropes of Neptune and Uranus are still slightly higher in

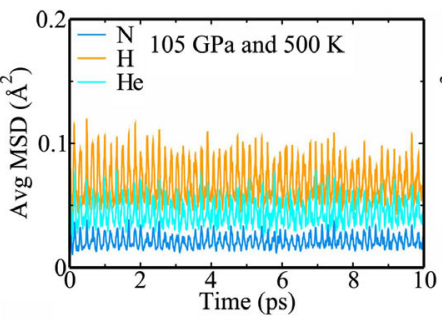

(a)

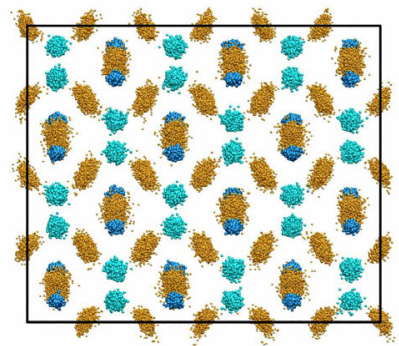

(e)

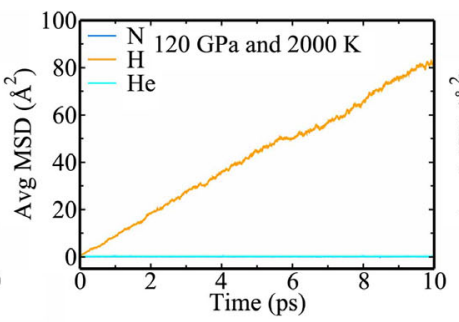

(b)

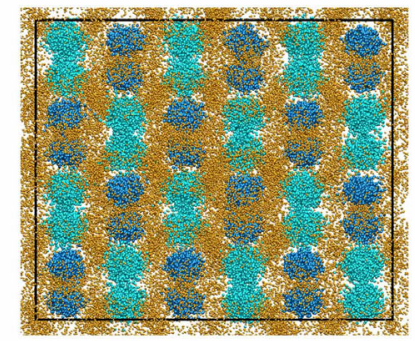

(f)

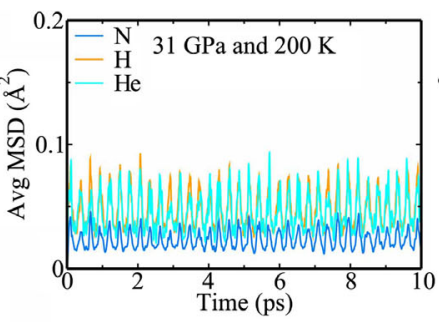

(c)

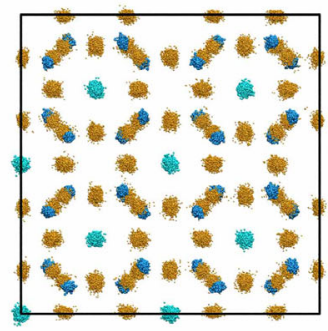

(g)

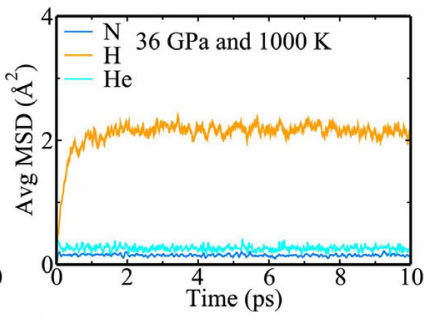

(d)

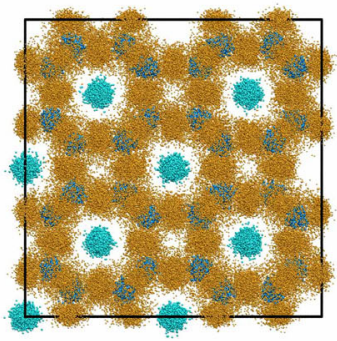

(h)

FIG. 2. Dynamical behavior of $\mathrm{H}$ (green) and He atoms (cyan) compared to $\mathrm{N}$ atoms (blue) in the helium ammonia compounds under

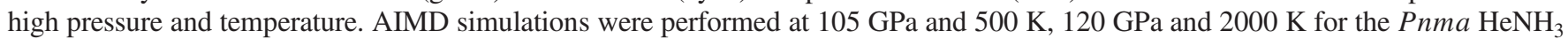
phase, and at $31 \mathrm{GPa}$ and $200 \mathrm{~K}, 36 \mathrm{GPa}$ and $1000 \mathrm{~K}$ for $\mathrm{I} 4 \mathrm{~cm} \mathrm{He}\left(\mathrm{NH}_{3}\right)_{2}$. (a)-(d) The averaged mean-squared displacements (MSD) at different temperatures. (e)-(h) Atomic trajectories from the simulations representing three different states of matter: the solid Pnma $\mathrm{HeNH}_{3}$ phase $(500 \mathrm{~K})$, the superionic phase $(2000 \mathrm{~K})$, the solid $\mathrm{I} 4 \mathrm{~cm} \mathrm{He}\left(\mathrm{NH}_{3}\right)_{2}$ phase $(200 \mathrm{~K})$, and the plastic phase (1000 K); the liquid phase is not shown. The $\mathrm{N}, \mathrm{H}$, and He atoms are respectively plotted with blue, green, and cyan. 
(a)

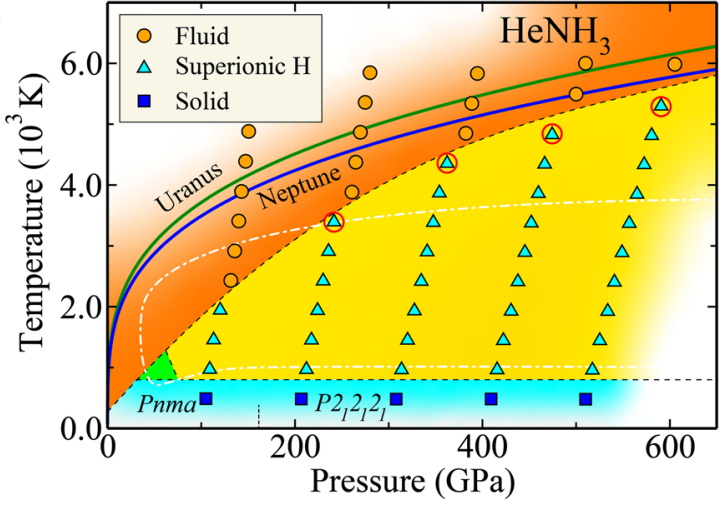

(b)

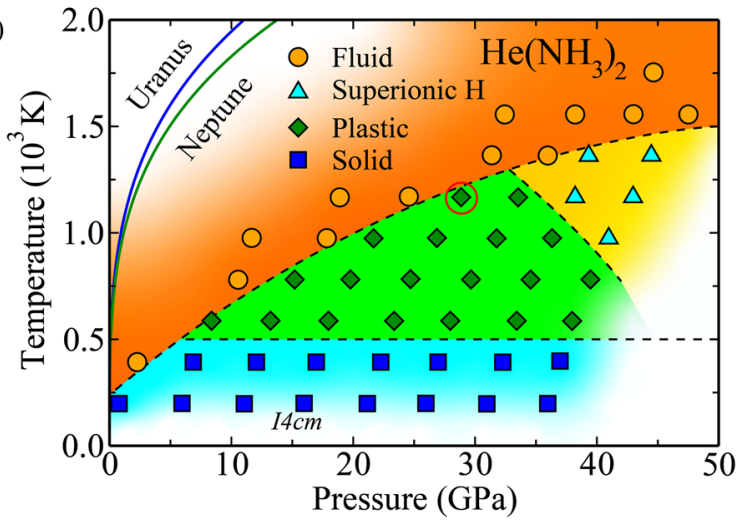

FIG. 3. Proposed phase diagram of $\mathrm{HeNH}_{3}$ (a) and $\mathrm{He}\left(\mathrm{NH}_{3}\right)_{2}$ (b) at high pressures obtained from structure searches and AIMD simulations. The simulations are marked with four different symbols: blue square, dark green diamond, cyan triangle, and orange circle represent the solid, plastic, superionic, and fluid states, respectively. Black dashed lines are fitted to the phase transition boundaries. The calculated isentrope for Uranus (Neptune) is shown as a thick dark green (dark blue) line. The white dash-dotted line covers the superionic phase in pure ammonia, taken from Redmer et al. [20]. Red solid circles mark some trajectories in which the superionic hydrogen or plastic phases coexist with diffusive helium, which has much smaller diffusion coefficients than superionic hydrogen near the phase boundaries.

temperature than the stability region of superionic helium ammonia compounds, it is possible that superionic helium ammonia might still form in interiors of other giant icy planets with different isentropes due to differences in mass, composition, or the distances to their main stars.

To explore the possible plastic state of the helium ammonia compounds at low pressures, we performed AIMD simulations for the host-guest $\mathrm{I} 4 \mathrm{~cm} \mathrm{He}\left(\mathrm{NH}_{3}\right)_{2}$ phase. As expected, we found that the $14 \mathrm{~cm}$ structure remains solid at low temperature, as shown in the MSD and atomic trajectory in Figs. 2(c) and 2(g). However, it enters a plastic state at around 10-40 GPa and 500-1200 K, in which hydrogen atoms rotate around the fixed nitrogen atoms, as shown in Fig. 3(b). One can find an atomic trajectory for such a plastic phase in Fig. 2(h), in which the three main distribution areas of hydrogen atoms surrounding the nitrogen atoms overlap due to the rotation of the ammonia molecules. The crucial signature of this type of plastic phase is a finite MSD with a plateau at a quite low value, as shown in Fig. 2(d). In the RDFs, the signature of hydrogen atoms in the plastic phase looks liquidlike, which is not very different from the superionic phase, as shown in Fig. S9(b) of the Supplemental Material [48].

On the boundary between the plastic and fluid phases we found in some trajectories that helium atoms become diffusive around the freely rotating ammonia molecules, as shown in Fig. 3(b) and Fig. S12 [48]. However, the pressures and temperatures of the plastic phases were lower than those of the superionic phase. Note that if we model the Pnma $\mathrm{HeNH}_{3}$ phase at lower pressures and temperatures, it also forms a plastic state, which has been outlined in Fig. 3(a). We find that both compounds exhibit a negative slope for the phase boundary between the plastic and superionic phases in the pressure-temperature phase diagram, which also occurs in pure water ice [25]. More details can be found in Figs. S13 and S14 in the Supplemental Material [48].

\section{DISCUSSION}

Hermann et al. [51] and French et al. [23] found that nuclear quantum effects significantly affect the water ice phase diagram at megabar pressures. The isotope effect may also affect the dynamical properties including the diffusion coefficient and vibrational spectrum. We therefore studied this in our helium ammonia system and carried out two independent AIMD simulations for the superionic states of $\mathrm{He}\left(\mathrm{NH}_{3}\right)_{2}$ and $\mathrm{He}\left(\mathrm{ND}_{3}\right)_{2}$ at around $2000 \mathrm{~K}$ and $100 \mathrm{GPa}$. We have estimated the nuclear quantum effects by comparing the vibrational density of states, as shown in Fig. 4. In the harmonic zero temperature phonon DOS, the high-frequency phonon peak decreases from about $3600 \mathrm{~cm}^{-1}$ for hydrogen to about $2600 \mathrm{~cm}^{-1}$ for deuterium, which is close to the ratio of $1 / \sqrt{2}$. As it was found that there is almost no charge transfer between helium and ammonia, such changes in the phonon frequencies are absent in the helium phonon DOS. When increasing the temperature to $2000 \mathrm{~K}$, the vibrational frequencies of both nitrogen and helium are almost identical in $\mathrm{He}\left(\mathrm{NH}_{3}\right)_{2}$ and $\mathrm{He}\left(\mathrm{ND}_{3}\right)_{2}$, and they also agree with their phonon DOS at zero temperature. However, both $\mathrm{H}$ and D have a nonzero DOS at zero frequency, which represents a finite diffusion coefficient. Their highest frequency modes are related to a redistribution of the $\mathrm{N}-\mathrm{H}(\mathrm{N}-\mathrm{D})$ bonds length, which represents the emergence of the superionic hydrogen/deuterium state. We calculated the diffusion coefficient from the averaged MSD, which decreased with increasing mass from $1.4 \times 10^{-8} \mathrm{~m}^{2} / \mathrm{s}$ for $\mathrm{H}$ to $0.88 \times$ $10^{-8} \mathrm{~m}^{2} / \mathrm{s}$ for D. The appearance of superionic phases in both $\mathrm{He}\left(\mathrm{NH}_{3}\right)_{2}$ and $\mathrm{He}\left(\mathrm{ND}_{3}\right)_{2}$ indicates that nuclear 


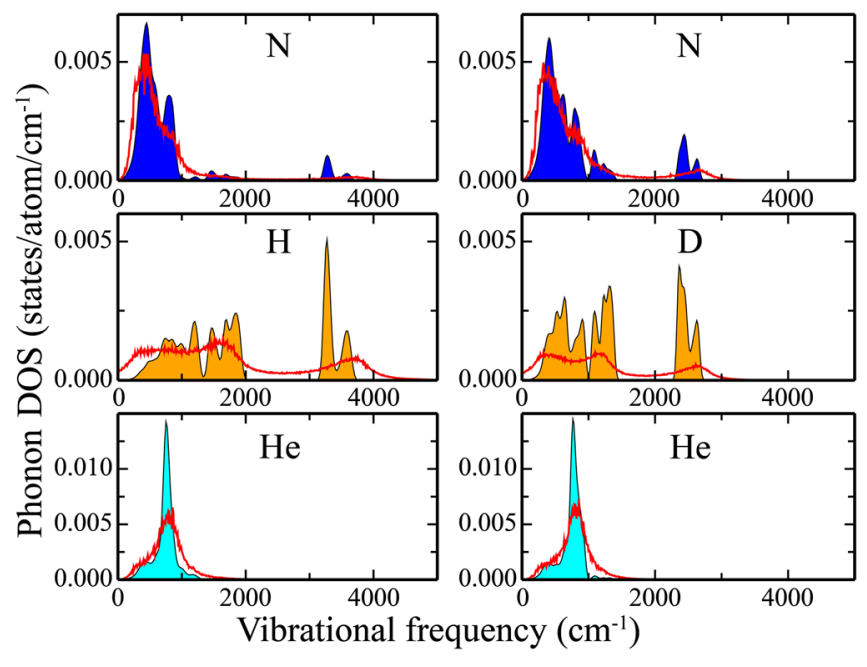

FIG. 4. Vibrational density of states (VDOS) for the Pnma phase $\mathrm{HeNH}_{3}$ (left) and $\mathrm{HeND}_{3}$ phase (right). Colored areas under black curves give the phonon DOS of $\mathrm{N}$ (blue), H/D, (orange) and $\mathrm{He}$ (cyan) from static phonon calculations at $0 \mathrm{~K}$. The red lines represent the vibrational DOS of each atomic species in the superionic phase at $100 \mathrm{GPa}+2000 \mathrm{~K}$, calculated from the Fourier transform of the velocity autocorrelation function. Note that the zero (nonzero) vibrational DOS at zero frequency is due to the fixed (diffusive) behavior of $\mathrm{O}, \mathrm{H} / \mathrm{D}$, and $\mathrm{He}$ in the superionic phase [22].

quantum effects do not affect the existence of the superionic state in the helium ammonia compounds, although we do not know whether the nuclear quantum effects affect the onset of the superionic state. We also compared the internal energy with the energy contribution from the nuclear quantum corrections, and found the influence to indeed be small, as listed in Table II in the Supplemental Material [48].

To cross-check our results of nuclear quantum effects, we have carried out path integral molecular dynamics (PIMD) with a colored noise approach, which has been successfully used in recent works by Ceriotti et al. [52,53] and Bronstein et al. [54]. We calculated the averaged meansquare displacement of the trajectory from such advanced PIMD simulation with eight beads, shown as Fig. S15 in Ref. [48]. Although the diffusion efficient is slightly reduced from $1.417 \times 10^{-8} \mathrm{~m}^{2} / \mathrm{s}$ (original AIMD) to $1.05 \times 10^{-8} \mathrm{~m}^{2} / \mathrm{s}$ (PIMD with colored noise approach), the conclusion remains the same that the superionic state of hydrogen atoms still exists in the Pnma $\mathrm{HeNH}_{3}$ under $120 \mathrm{GPa}$ and $2000 \mathrm{~K}$.

Our previous work showed that helium can become freely diffusive in a fixed water ice sublattice in helium water compounds and the diffusion coefficient of helium can even be higher than that of superionic hydrogen [13]. This can be explained because the helium atoms are weakly interacting with the ice network, and hence can move freely; on the other hand, protons are bound in oxygen with covalent bonds and require more energy to become diffusive. Strangely, such a superionic helium phase is absent in the helium ammonia compounds studied here. Although on the boundary of the superionic phase and fluid phase in helium ammonia compounds, some trajectories show that the helium atoms tend to be diffusive, the diffusion coefficient of helium in those cases is much lower than that of superionic hydrogen. This is very different from the case of the helium water system. To understand the reason for this difference, we carried out reduced density gradient (RDG) analysis [55] to investigate the interactions in the Pnma phase $\mathrm{HeNH}_{3}$ at $100 \mathrm{GPa}$, and compared the results with the $I 4_{1} m d \mathrm{He}_{2} \mathrm{H}_{2} \mathrm{O}$ phase at $10 \mathrm{GPa}$ and the $F d \overline{3} m \mathrm{He}_{2} \mathrm{H}_{2} \mathrm{O}$ phase at $100 \mathrm{GPa}$, as shown in Figs. 5(a)-5(c). The areas surrounded by solid magenta and dashed green lines represent the hydrogen bonding and $\mathrm{vdW}$ interactions in the different structures, which clearly show that the hydrogen bonding and vdW interactions in the Pnma phase of $\mathrm{HeNH}_{3}$ are more significant and discrete than in the $I 4_{1} m d \mathrm{He}_{2} \mathrm{H}_{2} \mathrm{O}$ phase at $10 \mathrm{GPa}$ and the $F d \overline{3} m \mathrm{He}_{2} \mathrm{H}_{2} \mathrm{O}$ phase at $100 \mathrm{GPa}$. We also analyzed the electron density around bond critical points (BCPs) $\rho\left(r_{\mathrm{BCP}}\right)$ based on the atom-in-molecule (AIM) theory [56]. The density $\rho\left(r_{\mathrm{BCP}}\right)$ reflects the relative bond strength, while its Laplacian is used to classify the chemical interactions: Laplacian values are negative for covalent bonds and positive for hydrogen bonds and $\mathrm{vdW}$ interactions. These data are available in Table III in the Supplemental Material [48]. We found that hydrogen bond interactions in the Pnma $\mathrm{HeNH}_{3}$ phase are quite significant while they are small in the $I 4_{1}$ md $\mathrm{He}_{2} \mathrm{H}_{2} \mathrm{O}$ phase and almost absent in $F d \overline{3} m \mathrm{He}_{2} \mathrm{H}_{2} \mathrm{O}$. Therefore, both RDG and $\mathrm{BCP}$ analysis show denser and more discrete interactions in Pnma $\mathrm{HeNH}_{3}$, which may hinder the diffusion of helium atoms. These indications inspired us to look into crystal structures and diffusion barrier in the following.

It is well known that vdW interactions are weaker than hydrogen bond interactions; therefore, the atoms with pure $\mathrm{vdW}$ interactions should diffuse more readily than the hydrogen bonded ones. However, for the superionic state of Pnma $\mathrm{HeNH}_{3}$, helium atoms remain almost fixed and the hydrogen atoms become freely diffusive. We therefore analyzed the structural environments of helium atoms in these three crystal structures, as shown in Figs. 5(d)-5(f). In the Pnma $\mathrm{HeNH}_{3}$ phase, each helium atom is enclosed within a distorted prismatic sublattice connected by $\mathrm{N}-\mathrm{H}$ covalent bonds and $\mathrm{H}-\mathrm{N}$ hydrogen bonds, as it roughly occupies the octahedral site of a perovskitelike lattice. Such a compact sublattice can place restrictions on the motions of the helium atoms and can reduce their tendency to diffusion. In the $F d \overline{3} m \mathrm{He}_{2} \mathrm{H}_{2} \mathrm{O}$ compounds at $100 \mathrm{GPa}$, the water ice sublattice forms hexagonal open channels with side length of $2.22 \AA$, and helium atoms can diffuse quite easily along these channels. Meanwhile, helium atoms stay relatively far from the ice sublattice, the closest 
(a)
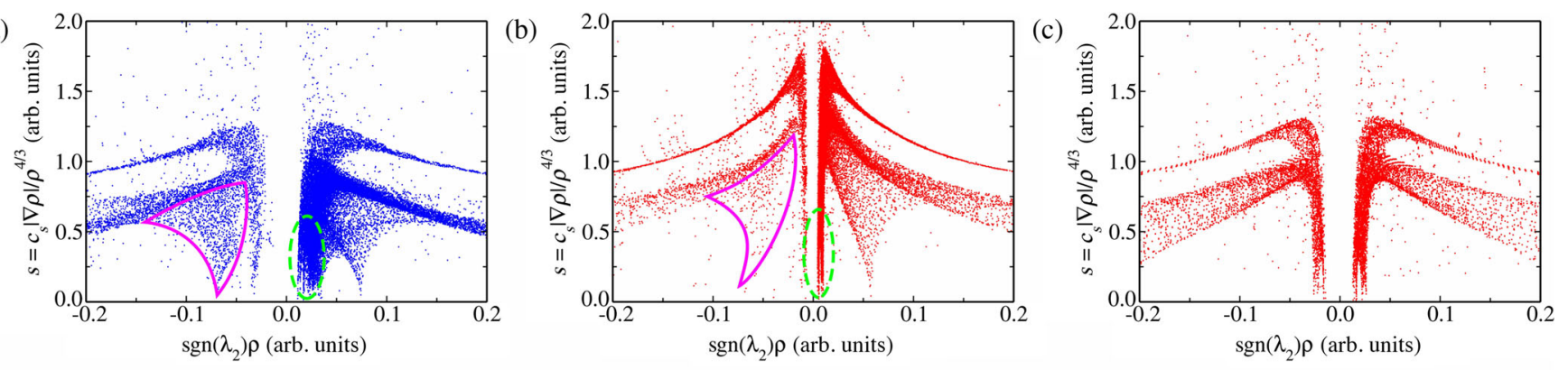

(d)

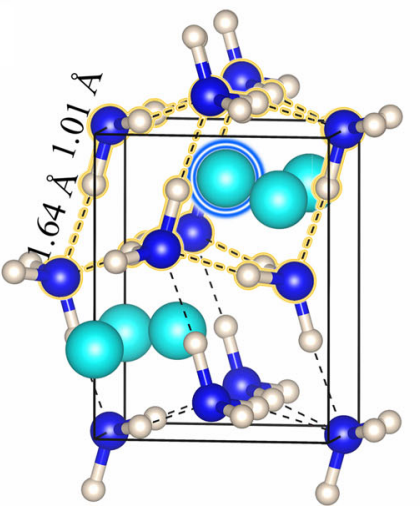

(e)

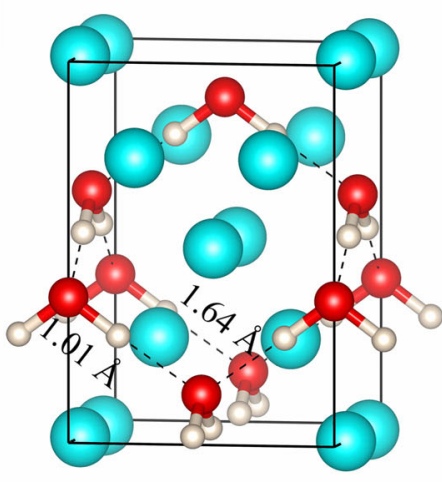

(f)

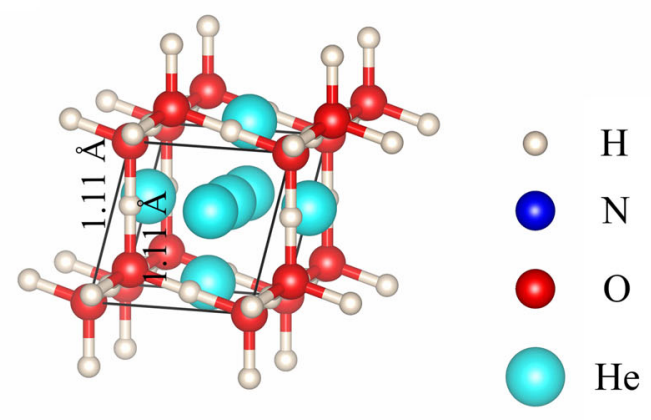

FIG. 5. RDG analysis and corresponding structural environment of helium atoms in the $\mathrm{HeNH}_{3}$ compound, compared with that in the helium water system. (a)-(c) Plots of RDG versus the electron density multiplied by the sign of the second Hessian eigenvalue for Pnma $\mathrm{HeNH}_{3}$ at $100 \mathrm{GPa}, I 4_{1} m d \mathrm{He}_{2} \mathrm{H}_{2} \mathrm{O}$ at $10 \mathrm{GPa}$, and $F d \overline{3} m \mathrm{He}_{2} \mathrm{H}_{2} \mathrm{O}$ at $100 \mathrm{GPa}$. The solid magenta (dashed green) line region represents the hydrogen bond (vdW interactions) in the crystalline. (d)-(f) Crystal structure of the abovementioned three structures. The covalent bond length and hydrogen bond length are marked nearby. In the $\mathrm{HeNH}_{3}$, blue highlighted helium atoms are enclosed in a light yellow highlighted distorted cubic sublattice, consisting of $\mathrm{N}-\mathrm{H}$ covalent bonds and $\mathrm{H}-\mathrm{N}$ hydrogen bonds.

He- $\mathrm{H}$ distance is about $1.81 \AA$. In the Pnma $\mathrm{HeNH}_{3}$ at $100 \mathrm{GPa}$ the closest He-H distance is about $1.65 \AA$, which is much shorter. These results are consistent with the conclusions from the charge density analysis. We also constructed the probability density function of the hydrogen atoms based on the atomic trajectories obtained from the simulations at $120 \mathrm{GPa}$ and $2000 \mathrm{~K}$, as shown in Fig. S16 [48], which illustrates the dynamical distribution of hydrogen atoms in the superionic $\mathrm{HeNH}_{3}$ Pnma phase. The helium atoms are retained in narrow and curved channels along the $\langle 100\rangle$ direction and there is also a high energy barrier between helium atoms, which further constrains the diffusion of the helium atoms. It should be noted that superionic hydrogen will not naturally open diffusion channels for helium since the hydrogen diffusion coefficient is much higher than that of helium: any instantaneous opening in the hydrogen-bonded ammonia network is too short-lived to allow large-scale motion of the helium atoms.

\section{CONCLUSION}

We systematically explored the helium ammonia system up to $500 \mathrm{GPa}$ and found three stoichiometries of stable compounds with eight new crystalline phases. Among them the Pnma phase $\mathrm{HeNH}_{3}$ and $P 2_{1} / c$ phases of $\mathrm{He}_{2} \mathrm{NH}_{3}$ feature a highly distorted perovskitelike structure in which ammonia is arranged in the octahedra, while half of the voids are empty and the other half are doubly occupied in the Pnma phase $\mathrm{HeNH}_{3}$ and all are doubly occupied in the $P 2_{1} / c$ phase of $\mathrm{He}_{2} \mathrm{NH}_{3}$. All compounds in the $\mathrm{He}\left(\mathrm{NH}_{3}\right)_{2}$ stoichiometry belong to a host-guest prototype in which all of the nitrogen atoms arrange in four- and eight-membered rings, and the helium atoms are distributed at the center of the eight-membered rings. In particular, the $14 \mathrm{~cm}$ phase becomes stable at pressures as low as $3 \mathrm{GPa}$ and its band gap has an abnormal pressure dependence. Furthermore, we carried out AIMD simulations to explore the dynamical properties of $\mathrm{HeNH}_{3}$ and proposed a new pressure-temperature phase diagram of helium ammonia compounds. This phase diagram features various thermally excited states-plasticity, superionicity, and the full melting line. While ground state phase transitions can be hindered by reaction kinetics [34], thermal excitations are in principle accessible with present-day high-pressure techniques applied to molecular systems up to the megabar regime: rotational disorder in plastic phases through quasielastic neutron scattering [57], superionicity via spectroscopy [27] or conductivity measurements [29], and melting via $\mathrm{X}$-ray diffraction [58].

We found a larger temperature range for superionic hydrogen in helium ammonia compounds than in pure ammonia, shifting the melting line higher and there- 
fore closer to icy planet isentropes, which may indicate a higher possibility for the existence of helium ammonia compounds under planetary conditions. Whereas near the boundary of the superionic and fluid phases in our helium ammonia phase diagram, the helium diffusion coefficient is much lower than that of hydrogen. Which is quite different from the previously studied helium-water system [13]. This is counterintuitive since the weak interaction between helium and the ammonia network suggests that helium could become mobile more easily than the tighter bound protons. We attribute this phenomenon to the compact ammonia sublattice held by covalent bonds and hydrogen bonds which enclose the helium atoms and form a highenergy barrier that reduces their tendency to diffusion, even if the hydrogen bond network itself rapidly changes as a consequence of the diffusive protons.

\section{METHODS}

\section{A. Crystal structure search}

We used a variable-composition structure-prediction algorithm, as implemented in a machine-learning accelerated crystal structure search [59], in which we combined the Bayesian optimization and a Gaussian process model to improve the search efficiency and diversity. This method has been successfully applied in many systems, including helium water compounds [13], T-graphene [60], and metal pentazolate salts [61], etc. We have performed extensive variable-composition searches on different pressures, such as 50,100, 300, and $500 \mathrm{GPa}$, as well as fixed stoichiometry $\left(\mathrm{He}: \mathrm{NH}_{3}=1: 2,1: 1\right.$, and $2: 1$, etc.) at these pressures (not every combination). The crystal structures obtained were cross-checked with results from the $a b$ initio random structure searching approach $[62,63]$, which came up with very similar results.

\section{B. Density functional theory calculations}

In this study DFT calculations were performed using the Vienna ab initio simulation package (VASP) [64], accompanied by the projector augmented-wave (PAW) method [65], with the normal version potentials, in which the $1 s^{2}$, $2 s^{2} 2 p^{3}$, and $1 s^{1}$ electrons are treated as valence electrons for $\mathrm{He}, \mathrm{N}$, and $\mathrm{H}$, and the generalized gradient approximation in the Perdew-Burke-Ernzerhof (PBE) exchange correlation functional [66]. To take the vdW interactions into account, we used DFT-D3 [49] corrections in all static structural calculations. Other charge-density-dependent vdW-DF corrections of Langreth and co-workers [67-69] (such as optB88 [70], optPBE [70], and rev-vdW-DF2 [71]), more advanced functionals (SCAN [72], HSE06 [73], PBE0 [50], and RPA [74]), and the hard version of pseudopotentials with smaller core radii are used to crosscheck the results; more details about the methods can be found in the Supplemental Material [48]. All of the structures presented were optimized by employing a plane wave cutoff of $720 \mathrm{eV}$ and dense Monkhorst-Pack Brillouin zone sampling grids with resolutions of $2 \pi \times 0.025 \AA^{-1}$, leading to ionic and cell optimizations with energy and force convergences better than $10^{-6} \mathrm{eV}$ and $0.002 \mathrm{eV} / \AA$, respectively. The CASTEP code [75] was used for the AIRSS searches, and similar convergence parameters as used in VASP were employed. Phonon calculations were performed with the phonopy package [76].

\section{Ab initio molecular dynamics simulations}

AIMD simulations based on the Born-Oppenheimer approximation implemented in VASP [59] were performed in $2 \times 3 \times 2$ supercells for Pnma $\mathrm{HeNH}_{3}$ (with 240 atoms) and $2 \times 2 \times 2$ supercell for $I 4 \mathrm{~cm} \mathrm{He}\left(\mathrm{NH}_{3}\right)_{2}$ (with 288 atoms), both with $\Gamma$-centered $k$ point in the Perdew-BurkeErnzerhof exchange correlation functional. A normal precision and a cutoff energy of $720 \mathrm{eV}$ were adopted to ensure energy convergence of better than $10^{-5} \mathrm{eV}$. The convergence tests of the $k$ mesh and the size effects are available in Table II in the Supplemental Material [48]. We adopted the canonical $N V T$ ensemble using a Nose-Hoover thermostat [77] with SMASS $=2$, lasting for 7 ps with a time step of $1 \mathrm{fs}$, and we allowed 2 ps for thermalization and then extracted data from the last $5 \mathrm{ps}$. Some trajectories were extended to 12 ps to check the stability of the simulations.

To obtain reliable diffusion coefficients, we carried out a 22 ps simulations under the same condition, compared with the superionic one under $100 \mathrm{GPa}$ and $2000 \mathrm{~K}$, as shown in Fig. S17 [48]. Also, the vdW corrections do not affect the diffusion behaviors, as shown in Fig. S18 [48].

We calculated the diffusion efficient from the averaged mean-square displacement and cross-checked by velocity autocorrelation function (VACF) by the formula below.

$$
\begin{aligned}
& \text { From MSD, }\left\langle\left[r_{i}(t)-r_{i}(0)\right]^{2}\right\rangle_{\alpha} \\
& =\frac{1}{N_{\alpha}} \sum_{i=1}^{N_{\alpha}}\left[r_{i}(t)-r_{i}(0)\right]^{2}, \\
& \begin{array}{c}
D_{\alpha}=\lim _{t \rightarrow \infty} \frac{1}{6 t}\left\langle\left[r_{i}(t)-r_{i}(0)\right]^{2}\right\rangle_{\alpha} . \\
\text { From VACF, }\left\langle\overrightarrow{v_{i}}(0) \cdot \overrightarrow{v_{i}}(\tau)\right\rangle_{\alpha} \\
=\frac{1}{N_{\alpha}} \sum_{i=1}^{N_{\alpha}} \frac{1}{t} \int_{0}^{t} v_{i}(t+\tau) \cdot v_{i}(\tau) d \tau, \\
D_{\alpha}=\frac{1}{3} \lim _{t \rightarrow \infty} \int_{0}^{t}\left\langle\overrightarrow{v_{i}}(0) \cdot \overrightarrow{v_{i}}(\tau)\right\rangle_{\alpha} d \tau .
\end{array}
\end{aligned}
$$

Furthermore, we can extract the vibrational density of states (VDOS) from VACF by Fourier transform:

$$
F_{\alpha}(\omega)=\lim _{t \rightarrow \infty} \frac{4 m_{\alpha}}{k_{B} T} \int_{0}^{t}\left\langle\overrightarrow{v_{i}}(0) \cdot \overrightarrow{v_{i}}(\tau)\right\rangle_{\alpha} \cos (2 \pi \omega t) d t .
$$

We formally normalized VDOS to the 3 frequency spectrum per species: 


$$
\int_{0}^{\infty} d \omega F_{\alpha}(\omega)=3 .
$$

Finally, the nuclear quantum corrections [23] can be expressed by

$$
\begin{aligned}
u_{v c}= & \frac{1}{m} \sum_{\alpha} N_{\alpha} \lim _{\omega \rightarrow \infty} \int_{0}^{\omega} d \omega F_{\alpha}(\omega) \\
& \times\left[h \omega\left(\frac{1}{2}+\frac{1}{\exp \left(h \omega / k_{B} T\right)-1}\right)-k_{B} T\right],
\end{aligned}
$$

where $m_{\alpha}$ is the mass of atoms for species $\alpha$ and $k_{B}$ is Boltzmann's constant and the total mass of atoms is defined as $m=\sum_{\alpha} N_{\alpha} m_{\alpha}$. We specified the time step $d \tau$ of $1 \mathrm{fs}$.

We cross-checked the nuclear quantum effect by path integral molecular dynamic with a colored noise method, implemented in the i-PI package [78].

\section{Charge density analysis}

The atoms-in-molecules (AIM) [56] and reduced density gradient (RDG) analyses [55] of the electron density $\rho(r)$ were performed by the CRITIC2 code [79].

\section{ACKNOWLEDGEMENTS}

J.S. gratefully acknowledges financial support from the National Key R\&D Program of China (Grant No. 2016YFA0300404), the National Natural Science Foundation of China (Grants No. 11574133, No. 11974162 , and No. 11834006), and the Fundamental Research Funds for the Central Universities. C. J. P. and R. J. N. acknowledge financial support from the Engineering and Physical Sciences Research Council (EPSRC) of the U. K. under Grants No. EP/G007489/2 (C.J.P.) and No. EP/P034616/1 (R. J. N.). C. J.P. also acknowledges financial support from EPSRC and the Royal Society through a Royal Society Wolfson Research Merit award. The calculations were carried out using supercomputers at the High Performance Computing Center of Collaborative Innovation Center of Advanced Microstructures, the high-performance supercomputing center of Nanjing University, "Tianhe-2" at NSCC-Guangzhou, and the CSD3 Peta4 CPU/KNL machine at the University of Cambridge.

C. J. P. is an author of the CASTEP code and receives royalty payments from its commercial sales by Dassualt Systemes.

[1] X. Dong et al., A Stable Compound of Helium and Sodium at High Pressure, Nat. Chem. 9, 440 (2017).
[2] B. Monserrat, M. Martinez-Canales, R. J. Needs, and C. J. Pickard, Helium-Iron Compounds at Terapascal Pressures, Phys. Rev. Lett. 121, 015301 (2018).

[3] J. Zhang, J. Lv, H. Li, X. Feng, C. Lu, S. A. T. Redfern, H. Liu, C. Chen, and Y. Ma, Rare Helium-Bearing Compound $\mathrm{FeO}_{2} \mathrm{He}$ Stabilized at Deep-Earth Conditions, Phys. Rev. Lett. 121, 255703 (2018).

[4] H. Gao, J. Sun, C. J. Pickard, and R. J. Needs, Prediction of Pressure-Induced Stabilization of Noble-Gas-Atom Compounds with Alkali Oxides and Alkali Sulfides, Phys. Rev. Mater. 3, 015002 (2019).

[5] Z. Liu, J. Botana, A. Hermann, S. Valdez, E. Zurek, D. Yan, H.-q. Lin, and M.-s. Miao, Reactivity of He with Ionic Compounds under High Pressure, Nat. Commun. 9, 951 (2018).

[6] P. Loubeyre, M. Jean-Louis, R. LeToullec, and L. Charon-Gérard, High Pressure Measurements of the He-Ne Binary Phase Diagram at 296 K: Evidence for the Stability of a Stoichiometric $\mathrm{NeHe}_{2}$ Solid, Phys. Rev. Lett. 70, 178 (1993).

[7] C. Cazorla, D. Errandonea, and E. Sola, High-Pressure Phases, Vibrational Properties, and Electronic Structure of $\mathrm{NeHe}_{2}$ and $\mathrm{ArHe}_{2}$ : A First-Principles Study, Phys. Rev. B 80, 064105 (2009).

[8] Y. Wang, J. Zhang, H. Liu, and G. Yang, Prediction of the Xe-He Binary Phase Diagram at High Pressures, Chem. Phys. Lett. 640, 115 (2015).

[9] W. L. Vos, L. W. Finger, R. J. Hemley, J. Z. Hu, H. K. Mao, and J. A. Schouten, A High-Pressure van der Waals Compound in Solid Nitrogen-Helium Mixtures, Nature (London) 358, 46 (1992).

[10] Y. Li, X. Feng, H. Liu, J. Hao, S. A. T. Redfern, W. Lei, D. Liu, and Y. Ma, Route to High-Energy Density Polymeric Nitrogen $t-N$ via He-N Compounds, Nat. Commun. 9, 1 (2018).

[11] H. Liu, Y. Yao, and D. D. Klug, Stable Structures of He and $\mathrm{H}_{2} \mathrm{O}$ at High Pressure, Phys. Rev. B 91, 014102 (2015).

[12] P. Teeratchanan and A. Hermann, Computational Phase Diagrams of Noble Gas Hydrates under Pressure, J. Chem. Phys. 143, 154507 (2015).

[13] C. Liu, H. Gao, Y. Wang, R. J. Needs, C. J. Pickard, J. Sun, H.-T. Wang, and D. Xing, Multiple Superionic States in Helium-Water Compounds, Nat. Phys. 15, 1065 (2019).

[14] Y. Bai et al., Electrostatic Force Driven Helium Insertion into Ammonia and Water Crystals under Pressure, Communications Series B, Chemistry and chemical engineering / Faculty of Sciences, University of Ankara 2, 1 (2019).

[15] I. A. Ryzhkin, Superionic Transition in Ice, Solid State Commun. 56, 57 (1985).

[16] P. Demontis, R. LeSar, and M. L. Klein, New High-Pressure Phases of Ice, Phys. Rev. Lett. 60, 2284 (1988).

[17] C. Cavazzoni et al., Superionic and Metallic States of Water and Ammonia at Giant Planet Conditions, Science 283, 44 (1999).

[18] N. Goldman, L. E. Fried, I.-F. W. Kuo, and C. J. Mundy, Bonding in the Superionic Phase of Water, Phys. Rev. Lett. 94, 217801 (2005).

[19] M. French, T. R. Mattsson, N. Nettelmann, and R. Redmer, Equation of State and Phase Diagram of Water at Ultrahigh 
Pressures as in Planetary Interiors, Phys. Rev. B 79, 054107 (2009).

[20] R. Redmer, T. R. Mattsson, N. Nettelmann, and M. French, The Phase Diagram of Water and the Magnetic Fields of Uranus and Neptune, Icarus 211, 798 (2011).

[21] H. F. Wilson, M. L. Wong, and B. Militzer, Superionic to Superionic Phase Change in Water: Consequences for the Interiors of Uranus and Neptune, Phys. Rev. Lett. 110, 151102 (2013).

[22] J. Sun, B. K. Clark, S. Torquato, and R. Car, The Phase Diagram of High-Pressure Superionic Ice, Nat. Commun. 6, 1 (2015).

[23] M. French, M. P. Desjarlais, and R. Redmer, Ab Initio Calculation of Thermodynamic Potentials and Entropies for Superionic Water, Phys. Rev. E 93, 022140 (2016).

[24] J.-A. Hernandez and R. Caracas, Superionic-Superionic Phase Transitions in Body-Centered Cubic $\mathrm{H}_{2} \mathrm{O}$ Ice, Phys. Rev. Lett. 117, 135503 (2016).

[25] J.-A. Hernandez and R. Caracas, Proton Dynamics and the Phase Diagram of Dense Water Ice, J. Chem. Phys. 148, 214501 (2018).

[26] A. F. Goncharov, N. Goldman, L. E. Fried, J. C. Crowhurst, I.-F. W. Kuo, C. J. Mundy, and J. M. Zaug Dynamic Ionization of Water under Extreme Conditions, Phys. Rev. Lett. 94, 125508 (2005).

[27] S. Ninet, F. Datchi, and A. M. Saitta, Proton Disorder and Superionicity in Hot Dense Ammonia Ice, Phys. Rev. Lett. 108, 165702 (2012).

[28] E. Sugimura, T. Komabayashi, K. Ohta, K. Hirose, Y. Ohishi, and L.S. Dubrovinsky, Experimental Evidence of Superionic Conduction in $\mathrm{H}_{2} \mathrm{O}$ Ice, J. Chem. Phys. 137, 194505 (2012).

[29] M. Millot, S. Hamel, J. R. Rygg, P. M. Celliers, G. W. Collins, F. Coppari, D. E. Fratanduono, R. Jeanloz, D. C. Swift, and J. H. Eggert, Experimental Evidence for Superionic Water Ice Using Shock Compression, Nat. Phys. 14, 297 (2018).

[30] M. Millot, F. Coppari, J. R. Rygg, A. C. Barrios, S. Hamel, D. C. Swift, and J. H. Eggert, Nanosecond X-Ray Diffraction of Shock-Compressed Superionic Water Ice, Nature (London) 569, 251 (2019).

[31] M. Bethkenhagen, D. Cebulla, R. Redmer, and S. Hamel, Superionic Phases of the 1:1 Water-Ammonia Mixture, J. Phys. Chem. A 119, 10582 (2015).

[32] X. Jiang, X. Wu, Z. Zheng, Y. Huang, and J. Zhao, Ionic and Superionic Phases in Ammonia Dihydrate $\mathrm{NH}_{3} \cdot 2 \mathrm{H}_{2} \mathrm{O}$ under High Pressure, Phys. Rev. B 95, 144104 (2017).

[33] V. N. Robinson, Y. Wang, Y. Ma, and A. Hermann, Stabilization of Ammonia-Rich Hydrate inside Icy Planets, Proc. Natl. Acad. Sci. U.S.A. 114, 9003 (2017).

[34] C. Liu et al., Topologically Frustrated Ionisation in a Water-Ammonia Ice Mixture, Nat. Commun. 8, 1 (2017).

[35] V. N. Robinson, M. Marqués, Y. Wang, Y. Ma, and A. Hermann, Novel Phases in Ammonia-Water Mixtures under Pressure, J. Chem. Phys. 149, 234501 (2018).

[36] V. N. Robinson and A. Hermann, Plastic and Superionic Phases in Ammonia-Water Mixtures at High Pressures and Temperatures, J. Phys. Condens. Matter 32 (2020).

[37] M. Bethkenhagen et al., Planetary Ices and the Linear Mixing Approximation, Astrophys. J. 848, 67 (2017).
[38] X. Song, K. Yin, Y. Wang, A. Hermann, H. Liu, J. Lv, Q. Li, C. Chen, and Y. Ma, Exotic Hydrogen Bonding in Compressed Ammonia Hydrides, J. Phys. Chem. Lett. 10, 2761 (2019).

[39] N. Kamaya et al., A Lithium Superionic Conductor, Nat. Mater. 10, 682 (2011).

[40] X. Wang, R. Xiao, H. Li, and L. Chen, Oxysulfide LiAlSO: A Lithium Superionic Conductor from First Principles, Phys. Rev. Lett. 118, 195901 (2017).

[41] X. He, Y. Zhu, and Y. Mo, Origin of Fast Ion Diffusion in Super-Ionic Conductors, Nat. Commun. 8, 1 (2017).

[42] H. Liu, X. Shi, F. Xu, L. Zhang, W. Zhang, L. Chen, Q. Li, C. Uher, T. Day, and G. J. Snyder, Copper Ion Liquid-like Thermoelectrics, Nat. Mater. 11, 422 (2012).

[43] W. Qiu, L. Xi, P. Wei, X. Ke, J. Yang, and W. Zhang, PartCrystalline Part-Liquid State and Rattling-like Thermal Damping in Materials with Chemical-Bond Hierarchy, Proc. Natl. Acad. Sci. U.S.A. 111, 15031 (2014).

[44] Y. Takii, K. Koga, and H. Tanaka, A Plastic Phase of Water from Computer Simulation, J. Chem. Phys. 128, 204501 (2008).

[45] J. L. Aragones and C. Vega, Plastic Crystal Phases of Simple Water Models, J. Chem. Phys. 130, 244504 (2009).

[46] L. E. Bove, S. Klotz, T. Strässle, M. Koza, J. Teixeira, and A. M. Saitta, Translational and Rotational Diffusion in Water in the Gigapascal Range, Phys. Rev. Lett. 111, 185901 (2013).

[47] B. Li et al., Colossal Barocaloric Effects in Plastic Crystals, Nature (London) 567, 506 (2019).

[48] See Supplemental Material at http://link.aps.org/ supplemental/10.1103/PhysRevX.10.021007 for more details about methods, crosscheck with different vdW corrections and hybrid functionals, crystal structures, electronic structures, phonon spectra, and dynamical properties, etc.

[49] S. Grimme, J. Antony, S. Ehrlich, and H. Krieg, A Consistent and Accurate Ab Initio Parametrization of Density Functional Dispersion Correction (DFT-D) for the 94 Elements $\mathrm{H}-\mathrm{Pu}$, J. Chem. Phys. 132, 154104 (2010).

[50] C. Adamo and V. Barone, Toward Reliable Density Functional Methods without Adjustable Parameters: The PBEO Model, J. Chem. Phys. 110, 6158 (1999).

[51] A. Hermann, N. W. Ashcroft, and R. Hoffmann, Isotopic Differentiation and Sublattice Melting in Dense Dynamic Ice, Phys. Rev. B 88, 214113 (2013).

[52] M. Ceriotti, G. Bussi, and M. Parrinello, Nuclear Quantum Effects in Solids Using a Colored-Noise Thermostat, Phys. Rev. Lett. 103, 030603 (2009).

[53] M. Ceriotti, M. Parrinello, T. E. Markland, and D. E. Manolopoulos, Efficient Stochastic Thermostatting of Path Integral Molecular Dynamics, J. Chem. Phys. 133, 124104 (2010).

[54] Y. Bronstein, P. Depondt, F. Finocchi, and A. M. Saitta, Quantum-Driven Phase Transition in Ice Described via an Efficient Langevin Approach, Phys. Rev. B 89, 214101 (2014).

[55] E. R. Johnson, S. Keinan, P. Mori-Sánchez, J. ContrerasGarcía, A. J. Cohen, and W. Yang, Revealing Noncovalent Interactions, J. Am. Chem. Soc. 132, 6498 (2010).

[56] R. F. W. Bader, A Quantum Theory of Molecular Structure and Its Applications, Chem. Rev. 91, 893 (1991). 
[57] S. Schaack, U. Ranieri, P. Depondt, R. Gaal, W. F. Kuhs, A. Falenty, P. Gillet, F. Finocchi, and L. E. Bove, Orientational Ordering, Locking-in, and Distortion of $\mathrm{CH}_{4}$ Molecules in Methane Hydrate III under High Pressure, J. Phys. Chem. C 122, 11159 (2018).

[58] G. Weck, F. Datchi, G. Garbarino, S. Ninet, J.-A. Queyroux, T. Plisson, M. Mezouar, and P. Loubeyre, Melting Curve and Liquid Structure of Nitrogen Probed by X-ray Diffraction to 120 GPa, Phys. Rev. Lett. 119, 235701 (2017).

[59] K. Xia et al., A Novel Superhard Tungsten Nitride Predicted by Machine-Learning Accelerated Crystal Structure Search, Sci. Bull. 63, 817 (2018).

[60] Q. Gu, D. Xing, and J. Sun, Superconducting Single-Layer T-Graphene and Novel Synthesis Routes, Chin. Phys. Lett. 36, 097401 (2019).

[61] K. Xia, J. Yuan, X. Zheng, C. Liu, H. Gao, Q. Wu, and J. Sun, Predictions on High-Power Trivalent Metal Pentazolate Salts, J. Phys. Chem. Lett. 10, 6166 (2019).

[62] C. J. Pickard and R. J. Needs, High-Pressure Phases of Silane, Phys. Rev. Lett. 97, 045504 (2006).

[63] C. J. Pickard and R. J. Needs, Ab Initio Random Structure Searching, J. Phys. Condens. Matter 23, 053201 (2011).

[64] G. Kresse and J. Furthmüller, Efficient Iterative Schemes for Ab Initio Total-Energy Calculations Using a Plane-Wave Basis Set, Phys. Rev. B 54, 11169 (1996).

[65] P. E. Blöchl, Projector Augmented-Wave Method, Phys. Rev. B 50, 17953 (1994).

[66] J. P. Perdew, J. A. Chevary, S. H. Vosko, K. A. Jackson, M. R. Pederson, D. J. Singh, and C. Fiolhais, Atoms, Molecules, Solids, and Surfaces: Applications of the Generalized Gradient Approximation for Exchange and Correlation, Phys. Rev. B 46, 6671 (1992).

[67] M. Dion, H. Rydberg, E. Schröder, D. C. Langreth, and B. I. Lundqvist, van der Waals Density Functional for General Geometries, Phys. Rev. Lett. 92, 246401 (2004).
[68] T. Thonhauser, V. R. Cooper, S. Li, A. Puzder, P. Hyldgaard, and D. C. Langreth, van der Waals Density Functional: SelfConsistent Potential and the Nature of the van der Waals Bond, Phys. Rev. B 76, 125112 (2007).

[69] K. Lee, É. D. Murray, L. Kong, B. I. Lundqvist, and D. C. Langreth, Higher-Accuracy van der Waals Density Functional, Phys. Rev. B 82, 081101 (2010).

[70] J. Klimeš, D. R. Bowler, and A. Michaelides, Chemical Accuracy for the van der Waals Density Functional, J. Phys. Condens. Matter 22, 022201 (2010).

[71] I. Hamada, van der Waals Density Functional Made Accurate, Phys. Rev. B 89, 121103 (2014).

[72] J. Sun, A. Ruzsinszky, and J. P. Perdew, Strongly Constrained and Appropriately Normed Semilocal Density Functional, Phys. Rev. Lett. 115, 036402 (2015).

[73] A. V. Krukau, O. A. Vydrov, A. F. Izmaylov, and G. E. Scuseria, Influence of the Exchange Screening Parameter on the Performance of Screened Hybrid Functionals, J. Chem. Phys. 125, 224106 (2006).

[74] M. Kaltak, J. Klimeš, and G. Kresse, Cubic Scaling Algorithm for the Random Phase Approximation: Self-Interstitials and Vacancies in Si, Phys. Rev. B 90, 054115 (2014).

[75] S. J. Clark et al., First Principles Methods Using CASTEP, Z. Kristallogr. 220, 567 (2009).

[76] A. Togo and I. Tanaka, First Principles Phonon Calculations in Materials Science, Scr. Mater. 108, 1 (2015).

[77] W. G. Hoover, Canonical Dynamics: Equilibrium PhaseSpace Distributions, Phys. Rev. A 31, 1695 (1985).

[78] M. Ceriotti, J. More, and D. E. Manolopoulos, i-PI: A Python Interface for Ab Initio Path Integral Molecular Dynamics Simulations, Comput. Phys. Commun. 185, 1019 (2014).

[79] A. Otero-de-la-Roza, E. R. Johnson, and V. Luaña, CRITIC2: A Program for Real-Space Analysis of Quantum Chemical Interactions in Solids, Comput. Phys. Commun. 185, 1007 (2014). 